\title{
Anomalous thickness dependence of the Hall effect in ultrathin Pb layers on $\mathrm{Si}(111)$
}

\author{
I. Vilfan, ${ }^{1,2, *}$ M. Henzler, ${ }^{1, \dagger}$ O. Pfennigstorf, ${ }^{1}$ and H. Pfnür ${ }^{1, \uparrow}$ \\ ${ }^{1}$ Institut für Festkörperphysik, Universität Hannover, Appelstrasse 2, D-30167 Hannover, Germany \\ ${ }^{2}$ J. Stefan Institute, Jamova 39, SI-1001 Ljubljana, Slovenia
}

(Received 29 May 2002; published 20 December 2002)

\begin{abstract}
The magnetoconductive properties of ultrathin $\mathrm{Pb}$ films deposited on $\mathrm{Si}(111)$ are measured and compared with density-functional electronic band-structure calculations on two-dimensional, free-standing, 1 to 8 monolayers thick $\mathrm{Pb}(111)$ slabs. A description with free-standing slabs is possible because it turned out that the Hall coefficient is independent of the substrate and of the crystalline order in the film. We show that the oscillations in sign of the Hall coefficient observed as a function of film thickness can be explained directly from the thickness dependent variations of the electronic band-structure at the Fermi energy.
\end{abstract}

DOI: 10.1103/PhysRevB.66.241306

PACS number(s): 73.50.Jt, 71.15.Mb, 73.20.At, 73.61.At

The electronic properties of crystalline bulk materials are well understood. However, as soon as the lateral dimension in any direction is reduced to a few atomic distances, each electron energy-band splits into a set of discrete subbands and the spatial confinement leads to quantum-size effects (QSE). The quantum-size confinement affects in particular the electron-transport properties. In an early theoretical approach Trivedi and Ashcroft ${ }^{1}$ considered transport of electrons in a metal film, confined in a two-dimensional potential well and scattered by impurities in the film and (or) by surface roughness. They predicted a saw-tooth-like variation of the conductivity with the film thickness with a period of half the Fermi wavelength. Such oscillations have not been observed in the experiment so far. Another source of QSEinduced conductivity oscillations is the layer-by-layer growth of, e.g., $\mathrm{Pb}$ films on $\mathrm{Si}(111)(7 \times 7)$ or $\mathrm{Si}(111)-\mathrm{Au}(6 \times 6)$ when the in-plane conductivity shows oscillations with the period of the interlayer spacing, associated with the degree of roughness of the surface. ${ }^{3,2}$ An even more spectacular, but so far unexplained, observation was the reversal of sign in the Hall coefficient $R_{H}$ of epitaxial $\mathrm{Pb}$ films on $\mathrm{Si}(111)$, grown on a surface that was precovered with about one monolayer (ML) of Ag which shows a $(\sqrt{3} \times \sqrt{3})$ reconstruction. ${ }^{4}$

In this report, we address the general issue of charge transport and Hall effect in ultrathin metallic layers both theoretically and experimentally. We focus on $\mathrm{Pb}$ films deposited on $\mathrm{Si}(111)$, although the conclusions should be generally valid for ultrathin metallic films in the region of QSE. In order to clarify the underlying mechanisms, we combined in our study theoretical investigations by density-functional calculations with measurements of conductivity and of magnetoconduction of ultrathin $\mathrm{Pb}$ films on a $(7 \times 7)$ reconstructed $\mathrm{Si}(111)$ substrate.

$\mathrm{Pb}$ films with a thickness between 1 and 12 monolayers (ML) have been deposited in UHV on a well annealed $\operatorname{Si}(111)-(7 \times 7)$ substrate at low temperatures $(15$ to $25 \mathrm{~K})$. The thickness, crystalline order and defects have been monitored with high-resolution low-energy electron diffraction (SPA-LEED). ${ }^{5} 4$ Mo contacts have been predeposited for the measurements. After the pseudomorphic first $\mathrm{Pb}$ layer, up to $4 \mathrm{~Pb}$ layers were amorphous, whereas thicker layers always turned out to be crystalline. By varying temperature and thus the growth conditions, the influence of order on the conduc- tive and magnetoconductive properties was tested. For details on film preparation and ordering in the films, see Ref. 6.

Before starting the Hall effect measurement the films have been annealed close to temperature where the decrease of conductance indicated a breakup of the film into noncontiguous islands. Even after annealing, films with a thickness $d$ $<4$ ML were strongly disordered whereas thicker films grew epitaxially with the lattice constant of bulk $\mathrm{Pb}$. While the conductance measurements, which provide information on the charge-carrier scattering, were to some extent sensitive to the degree of order, the oscillatory behavior of the Hall coefficient turned out to be remarkably insensitive. Therefore, it seems to be justified as a first approach to compare the experimental results of the Hall coefficient with those obtained theoretically for free-standing $\mathrm{Pb}$ slabs. Indeed, as we will show below, such calculations are able to identify the basic origin of the oscillations.

Conductance and magnetoconductance measurements were performed in a dc magnetic field up to $4 \mathrm{~T}$ at various temperatures down to about $7 \mathrm{~K}$. In the van-der-Pauw arrangement for conductance ${ }^{7}$ it has been checked that the magnetoconductance was completely symmetric. In the contact arrangement for Hall effect there was a sizable contribution from magnetoconductance due to deviations from a perfect quadratic arrangements of the contacts. Therefore, the asymmetry was taken as the contribution of the Hall effect to the measured voltage. The Hall coefficient has been derived from this asymmetry at $\pm 4 \mathrm{~T}$.

In the model calculations we treat the $\mathrm{Pb}$ layers as freestanding slabs and neglect, as mentioned, the effect of the substrate. Within the model we calculate, as a function of layer thickness, the densities of states at the Fermi energies, Fermi velocities, and effective-mass tensors. From these quantities, together with the experimental data for the conductivity $\sigma_{0}^{\exp }$, the elastic scattering times are calculated and the Hall coefficient $R_{H}$ is derived.

The Hall coefficient in the low-field limit is

$$
R_{H}=\frac{\sigma_{H}}{\sigma_{0}^{2}},
$$

where $\sigma_{0}$ and $\sigma_{H}$ are the electrical and Hall conductivities of a two-dimensional slab. Adapting the three-dimensional 
expressions $^{8}$ to two dimensions we have

$$
\sigma_{0}=\frac{e^{2}}{V \hbar^{2}} \sum_{n, k} \tau_{n}(k)\left[\nabla_{k} \epsilon_{n}(k)\right]^{2}\left(-\frac{\partial f(\epsilon)}{\partial \epsilon}\right)
$$

and

$$
\begin{aligned}
\sigma_{H}= & \frac{e^{3}}{V} \sum_{n, k} \tau_{n}(k)^{2} \vec{v}_{n}(k)\left[\operatorname{Tr}\left(\mathbf{M}^{-1}\right)-\mathbf{M}^{-1}\right] \vec{v}_{n}(k) \\
& \times\left(-\frac{\partial f(\boldsymbol{\epsilon})}{\partial \boldsymbol{\epsilon}}\right) .
\end{aligned}
$$

$\mathbf{M}^{-1}$ is the inverse mass tensor with the elements

$$
\left(\mathbf{M}^{-1}\right)_{i, j}=\frac{1}{\hbar^{2}} \frac{\partial^{2} \epsilon_{n}(k)}{\partial k_{i} \partial k_{j}}
$$

$V$ the slab volume, $\vec{v}_{n}(k)=(1 / \hbar) \vec{\nabla} \epsilon_{n}(k)$ the electron group velocity, $\epsilon_{n}(k)$ the energy of an electron with momentum $k$ in the subband $n$, and $f$ the Fermi function. The sums in Eqs. (2) and (3) run over the wave vectors $k$ in the twodimensional hexagonal Brillouin zone and over the electron subband index $n$. (Each atomic electron level splits into $d$ two-dimensional electron subbands, where $d$ is the number of monolayers in the slab.) The spin degeneracy is included in the prefactors. As will be seen below, the relaxation time $\tau_{n}(k)$ of thin slabs at low temperatures is dominated by elastic scattering on the thickness fluctuations of the $\mathrm{Pb}$ film (roughness of the interface to the substrate). Therefore, we will take the limit of short-wavelength surface height fluctuations (with their correlation length $\xi \ll 1 / k_{F}, k_{F}$ being the Fermi wave vector). In this case the relaxation time is $k$ independent. ${ }^{9}$ Since no detailed information on the scattering mechanisms and on the interface structure is available, we assume that $\tau$ is also independent of the subband index $n$. Then, $R_{H}$ is independent of $\tau$ and is determined solely by the electron band structure at the Fermi energy $\epsilon_{F}$. The electronic band-structure calculations were performed on relaxed 1 to 8 monolayers thick (111) oriented $\mathrm{Pb}$ slabs with the in-plane lattice constant $a=3.50 \AA$, separated by $\approx 10 \AA$ of vacuum. The electron band energies and the total energy have been calculated using the full-potential linearized augmented plane-wave method within the WIEN2K code ${ }^{10}$ in the local-density approximation. ${ }^{11} \mathrm{~A} \mathrm{~Pb}$ muffin-tin radius of 2.6 a.u. and a tetrahedral mesh of $623 k$-points in the irreducible part of the full Brillouin zone were used in the self-consistent electronic structure calculations. The kinetic-energy cutoff was set to $E_{\max }^{\mathrm{wf}}=7.3 \mathrm{Ry}$ and the plane-wave expansion cutoff to $E_{\max }^{\mathrm{pw}}=100 \mathrm{Ry}$. Later, in the magnetoconduction calculations, we used about $4000 k$-points in the irreducible part of the two-dimensional Brillouin zone.

Several electron subbands cross the Fermi energy and there are several Fermi lines in the two-dimensional Brillouin zone. As typical examples, Fig. 1 shows the Fermi lines of 3 and $6 \mathrm{ML}$ thick slabs. The number of Fermi lines is an increasing function of the slab thickness. Only electrons close to the Fermi lines take part in the transport and $\sigma_{0} / \tau$ and $\sigma_{H} / \tau^{2}$ are obtained after numerical integration of ex-

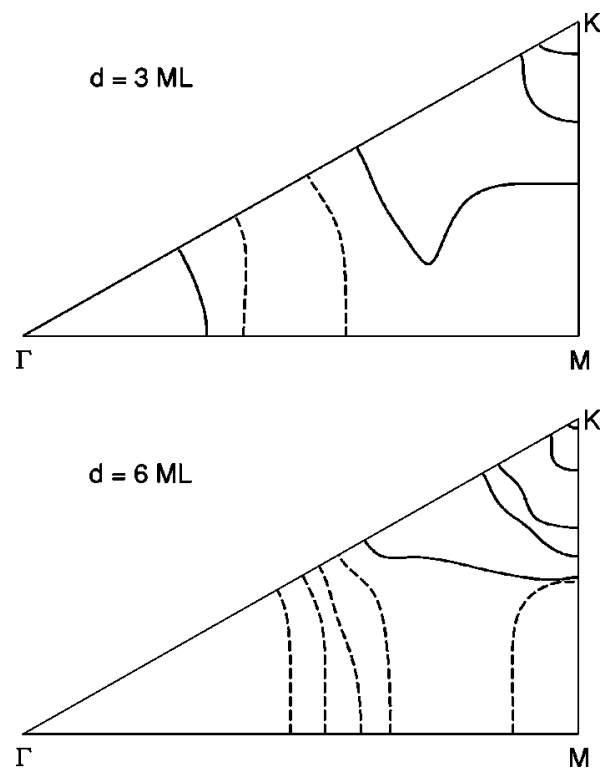

FIG. 1. Fermi lines of relaxed 3 and 6 ML thick free-standing $\mathrm{Pb}$ slabs. The solid lines encircle electron and dashed lines hole pockets.

pressions (2) and (3) along the Fermi lines. Whereas $\sigma_{0}$ is always proportional to the density of states at the Fermi lines, $\sigma_{H}$ of a subband is negative if the Fermi line makes a closed loop around occupied states (electrons) and positive if the loop encircles empty states (holes) in a subband. It is the balance between the number of electron and hole states at $\epsilon_{F}$ together with their effective masses which determines the sign of $R_{H}$. As a consequence, $R_{H}$ is sensitive to the details of the band structure.

Figure 2 shows the experimental conductivities $\sigma_{0}^{\exp }$ of annealed $\mathrm{Pb}$ films on $\mathrm{Si}(111)-(7 \times 7)$ together with the data

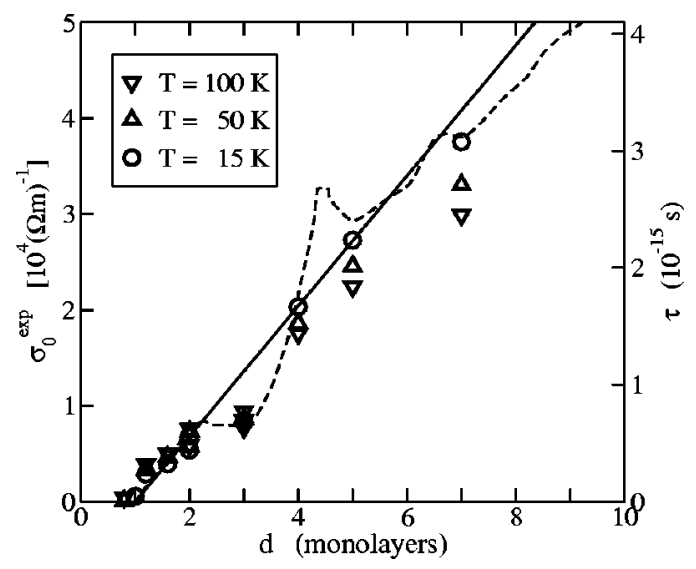

FIG. 2. Experimental conductivity $\sigma_{0}$ and the calculated average relaxation time $\tau$. The symbols are for annealed $\mathrm{Pb}$ films on $\mathrm{Si}(111)-(7 \times 7)$, measured at three different temperatures, the dashed line is for epitaxial $\mathrm{Pb}$ on $\mathrm{Si}(111)-\operatorname{Ag}(\sqrt{3} \times \sqrt{3})$ at $T$ $=20 \mathrm{~K}$ from Ref. 4 and the solid line is a linear fit to the lowtemperature data for $d<6 \mathrm{ML}$. The main process limiting the conductivity and the relaxation time in $\mathrm{Pb}$ ultrathin films is scattering on the interface fluctuations, the disorder within the film plays only a minor role. 
of epitaxial $\mathrm{Pb}$ on $\mathrm{Si}(111)$ covered with $\mathrm{Ag} .{ }^{4}$ Three properties of these data indicate that interface and (or) surface scattering is the dominant mechanism of (elastic) electron scattering in this system. First, only a weak temperature dependence of $\sigma_{0}^{\exp }$ is found below $100 \mathrm{~K}$. Second, there is only a weak dependence of conductivity on crystallinity and order in the films. Annealing of amorphous films grown at $15 \mathrm{~K}$ resulted in an increase of conductivity, but this increase never exceeded a factor of 2.5. Third, the roughly linear thickness dependence of $\sigma_{0}^{\exp }$ is again consistent with the assumption just made that the dominant scattering mechanism in these ultrathin films is diffuse scattering at interface fluctuations. ${ }^{9,12}$ For thicker samples the scattering at imperfections within the film becomes noticeable, and the conductivity starts to deviate from the linear behavior and eventually saturates in the bulk value, where the scattering on lattice defects and at higher temperatures also on phonons limit the conductivity.

We measured $\sigma_{0}^{\exp }$ for arbitrary film thickness $d$. At noninteger thicknesses the film surface is covered with islands and their borders (i.e., steps) act as scattering centers for the electrons. Therefore, one would expect a dip in $\sigma_{0}^{\text {exp }}$ for noninteger thicknesses. However, a small effect of this kind has only been observed at $d>12 \mathrm{ML}$. This suggests that the typical island sizes exceed the charge-carrier mean free path for very thin layers and electron scattering at the vacuum interface is not important for films with $d<10$.

Clearly seen is an offset in the conductivity towards $d$ $=1 \mathrm{ML}$. The onset of conductivity due to percolation of the evaporated $\mathrm{Pb}$ at a surface temperature of $15 \mathrm{~K}$ was found to be at $0.8 \mathrm{ML}$. The very low conductivity of this first monolayer indicates that the main transport mechanism in this layer is hopping between localized states. Indeed, the conductivity shows a temperature-activated behavior for $d$ $=1 \mathrm{ML}^{6}{ }^{6}$ The first $\mathrm{Pb}$ monolayer on $\operatorname{Si}(111)-(7 \times 7)$ has the periodicity of the substrate ${ }^{5}$ and may incorporate the adatoms of the $(7 \times 7)$ substrate. This might be the reason for the very low conductivity and for the localization of charge carriers.

The close agreement of the conductivity data obtained in our measurements with those of Ref. 4 is not at all selfevident, since our measurements have been carried out on $\mathrm{Pb}$ films that are expected to be much more disordered than those grown epitaxially on $\operatorname{Si}(111)-\operatorname{Ag}(\sqrt{3} \times \sqrt{3})$. Since the thickness dependence is almost the same for $\mathrm{Pb}$ films prepared on both substrates, we must conclude that interface scattering plays the same dominant role on both substrates. This means on the other hand that the degree of order in these ultrathin films is of secondary importance for electronic transport. We also note that the Ag interface layer obviously does not contribute to the measured conductance.

In contrast to the experimentally measured $\sigma_{0}^{\exp }$, the calculations of $\sigma_{0} / \tau$ and $\sigma_{H} / \tau^{2}$ were performed only for integer $d$. Surprisingly, $\sigma_{0} / \tau$ turned out to be almost independent of the slab thickness in the investigated range between 2 and $8 \mathrm{ML}$,

$$
\frac{\sigma_{0}}{\tau} \approx 1.1 \times 10^{21}(\Omega \mathrm{m} \mathrm{s})^{-1} .
$$

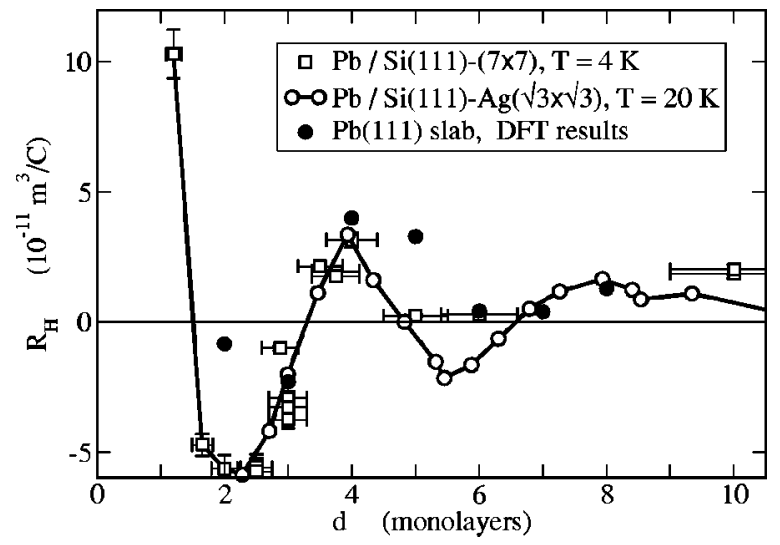

FIG. 3. Quantum-size induced oscillations of the Hall coefficient with the thickness $d$. Solid circles: calculations for a freestanding $\mathrm{Pb}$ slab; open symbols: experimental values for $\mathrm{Pb}$ films on two different $\mathrm{Si}(111)$ substrates (open circles are from Ref. 4) Unless explicitly indicated, the experimental error bars are of the size of the symbols. The estimated error bars of calculated $R_{H}$ are $\pm 1 \times 10^{-11} \mathrm{~m}^{3} / \mathrm{C}$ and come mainly from uncertainty in the broadening of the energy levels due to scattering. The solid line is a guide for the eye through the experimental points.

With increasing number of monolayers the number of subbands increases and thus the conductance but not the ratio $\sigma_{0} / \tau$. This means that the contributions of newly emerging subbands to $\sigma_{0} / \tau$ as a function of layer thickness are very similar. Equation (5) implies that $\tau$ is proportional to $\sigma_{0}$. Using the experimental values for $\sigma_{0}$, we determined $\tau$, also shown in Fig. 2.

It is interesting to look at the charge-carrier mean free path $\lambda=v_{F} \tau$. From the bandstructure we get the Fermi velocity $v_{F} \sim 1.2 \times 10^{6} \mathrm{~m} / \mathrm{s}$ and, using the values for $\tau$ from Fig. 2, we see that $\lambda$ is of the order 1 to 2 film thicknesses. It is therefore tempting to say that the electrons scatter on the film surfaces. However, the charge carriers scatter only on imperfections (steps, misplaced atoms etc.) on the surfaces and not on a perfectly flat surface. For $d=1$ ML the mean free path is shorter than the lattice spacing and the wavevector description of the electron states breaks down.

The relaxation-time causes broadening of the electron energy levels in the vicinity of the Fermi energy. Therefore, $R_{H}$ was calculated as $\left\langle\sigma_{0} / \tau\right\rangle /\left\langle\sigma_{H} / \tau^{2}\right\rangle$ where \langle\rangle is an average over the Gaussian distribution with the half-width $\delta \sim \hbar / \tau$.

The experimental results (open symbols) of the Hall coefficient are compared in Fig. 3 with those calculated (solid circles). Whereas the calculations have been done for perfectly periodic slabs without substrate, the measurements refer to epitaxial films with different degrees of disorder. Clearly seen are the anomalous oscillations of $R_{H}$ as a function of film thickness $d$ for both sets of experimental data. Our experimental results (squares) agree very well within the experimental error with the results of Ref. 4 (open circles in Fig. 3). After having found close agreement for the data of electrical conductance, the agreement of the results for $R_{H}$ are not surprising, since the disorder within the film plays a negligible role. On the other hand, this result justifies our assumption of scattering times independent of $k$ and $n . R_{H}$, calculated in this paper without any adjustable parameter, 
reproduces the oscillating behavior seen in the experiment semiquantitatively and demonstrates that the main reason for this behavior are the thickness dependent changes in the band structure. The deviations at $d=2$ and $d=5$ may be a consequence of the approximation of equal relaxation times for all subbands. Especially in the case $d=2$ also the $7 \times 7$ periodicity of the substrate, which was neglected in the slab calculation, might play a role. Our calculations also show that $\sigma_{0} / \tau$ is a slowly varying function of energy. Therefore, it is not very sensitive to the exact location of the Fermi energy within the band structure, and the conductance increases smoothly with film thickness, and with only small variations during deposition. In contrast, the calculated $\sigma_{H} / \tau^{2}$ is a strongly energy-dependent function and most of the energy dependence of $R_{H}$ comes from $\sigma_{H} / \tau^{2}$, therefore $R_{H}$ is sensitive to averaging.

To conclude, we have shown that only a quantitative band-structure analysis, done here with DFT, allows an understanding of the anomalous magnetoconductive properties of ultrathin $\mathrm{Pb}$ films. Oscillations in $R_{H}$ as a function of film thickness are the consequence of competing - and to a large extent compensating - contributions of electrons from different subbands and cannot be explained with any model which does not take into account the details of the electronic band structure. The relaxation time and thus the interface fluctuations play a subordinate role, they affect $R_{H}$ through the width of the distribution function and through the subband dependence of the relaxation time, which was neglected in this paper. Although our calculations with free-standing films already show all qualitative features observed in experiment, it is obvious that this method could be further refined by explicitly taking into account the underlying substrate. This might help to understand the specific role of the metalsemiconductor interface considered here. Generally, this interplay between calculations and experiments is of primary importance since inner interfaces cannot be varied easily in experiments. In this context, also the open problem of the very low conductivity and the high $R_{H}$ of the first monolayer may be solvable. This involves most likely hopping conduction between localized states in the $\mathrm{Pb}$ film on reconstructed $\mathrm{Si}(111)$ substrate.

The authors are grateful to Professor Matthias Scheffler for interesting discussions and Professor Peter Saalfrank for sending us his results prior to publication. I.V. would like to acknowledge the hospitality of the Institut für Festkörperphysik in Hannover. This work was supported in part by the Deutsche Forschungsgemeinschaft.
*Electronic address: igor. vilfan@ijs. si

†Electronic address: henzler@fkp. uni-hannover. de

Electronic address: pfnuer@fkp. uni-hannover. de

${ }^{1}$ N. Trivedi and N.W. Ashcroft, Phys. Rev. B 38, 12298 (1988).

${ }^{2}$ G. Palasantzas and J.Th.M. De Hosson, Phys. Rev. B 63, 125404 (2001).

${ }^{3}$ M. Jalochowski and E. Bauer, Phys. Rev. B 37, 8622 (1988); M. Jalochowski and E. Bauer, ibid. 38, 5272 (1988); M. Jalochowski, E. Bauer, H. Knoppe, and G. Lilienkamp, ibid. 45, 13607 (1992).

${ }^{4}$ M. Jalochowski, M. Hoffmann, and E. Bauer, Phys. Rev. Lett. 76, 4227 (1996).

${ }^{5}$ A. Petkova, J. Wollschläger, H.L. Günter, and M. Henzler, Surf.
Sci. 482-485, 922 (2001); 471, 11 (2001).

${ }^{6}$ O. Pfennigstorf, A. Petkova, H.L. Günter, and M. Henzler, Phys. Rev. B 65, 45412 (2002).

${ }^{7}$ L.J. van der Pauw, Philips Res. Rep. 13, 1 (1958).

${ }^{8}$ W.W. Schulz, P.B. Allen, and N. Trivedi, Phys. Rev. B 45, 10886 (1992).

${ }^{9}$ D. Calecki, Phys. Rev. B 42, 6906 (1990).

${ }^{10}$ P. Blaha, K. Schwarz, G. K. H. Madsen, D. Kvasnicka and J. Luitz, WIEN2K, a full potential LAPW package, K. Schwarz, TU Vienna, 2001.

${ }^{11}$ J.P. Perdew and Y. Wang, Phys. Rev. B 45, 13244 (1992).

${ }^{12}$ H.E. Camblong and P.M. Levy, Phys. Rev. B 60, 15782 (1999). 